\title{
Time-Temperature-Shift Principle and Stepped Isothermal Method applied on ETFE-Foils
}

\author{
Jonas Hornig ${ }^{*}$, Karsten Moritz ${ }^{* *}$, Benjamin König ${ }^{\dagger}$
}

* Ingenieurgruppe Bauen, Besselstraße 16,68219 Mannheim, Germany, e-mail:
jonas.hornig@ingenieurgruppe-bauen.de, web page: http://www.ingenieurgruppe-bauen.de
** Taiyo Europe GmbH, Mühlweg 2, Mühlweg 2, 82054 Sauerlach, Germany, e-mail:
k.moritz@taiyo-europe.com, web page: https://taiyo-europe.com/de/
† Württemberger Str. 21, 89150 Laichingen, Germany, e-mail: benjaminkoenig@live.de

\begin{abstract}
The application of the time-temperature shift (TTS) principle on plastic materials, especially viscoelastic polymers, has been known since a long time. This principle describes the influences of the parameters time (embodied by the strain rate) and temperature on the polymer's mechanical behaviour (stress, strain, stiffness, strength values). In case of polymers that allow the application of the TTS both parameters obviously have an inversely proportional influence on the molecular chains and, therefore, on the mechanical properties of the material. The TTS allows the conversion of both parameters, but also the prediction of the mechanical behaviour in ranges that haven't been tested. In case of ETFEfoils the TTS is the reason for their applicability in the building practice that means under snow loads with low strain rates and low temperatures and under wind loads at high strain rates and high temperatures. In both load case scenarios the materials stiffness and strength values keep in acceptable ranges. The TTS principle has been applied on ETFE foils in 2007 the first time [1].

The evaluation of an extensive parameter study in form of uniaxial short-term tensile tests was then used to derive a master curve and to formulate an ARRHENIUS-function for the mathematical description of the TTS of ETFE foils [2]. This function allowed the first time to predict the mechanical behaviour of ETFE foils for combinations of strain rates and temperatures that haven't been investigated before.

With the stepped isotherm method (SIM), an additional approach is available that allows the derivation of the TTS-function of such polymers, too. The method applied is based on uniaxial short-term creep tests at temperatures that increase step by step. From the preceding time-dependent expansion curves a master curve has been derived. Shifting the master curve along the time axis enabled the prediction of the long-term behaviour of the investigated ETFE-foils. The time-dependent strain curves determined with this method have been validated by uniaxial long-term creep tests. [3]
\end{abstract}

\section{REFERENCES}

[1] K. Moritz, ETFE-Foil as Structural Element, Dissertation, TU Munich, 2007

[2] B. König, Experimental Determination of the Time-Temperature-Shift of ETFE-Film with Short-Time Tensile-Tests, Master Thesis, FH Munich, 2012

[3] J. Hornig, Experimental investigations on the creep behavior of ETFE foils using the stepped isothermal method, 2020 (not published) 